\title{
Quasiparticle relaxation rates in a spatially inhomogeneous superconductor
}

\author{
A.A. Golubov ${ }^{1}$ and E.P. Houwman \\ University of Twente, Department of Applied Physics, 217, 7500 AE Enschede, The Netherlands
}

Received 10 October 1992

\begin{abstract}
Effective quasi-particle relaxation rates in reduced gap regions of a dirty superconductor ( $\mathrm{S}$ ) at low temperatures are calculated from microscopic theory. Gap reduction in $\mathrm{S}$ caused by a proximity layer $\left(\mathrm{S}^{\prime}\right)$ with lower critical temperature is modeled by an effective trapping layer with zero gap and an effective thickness $L_{\mathrm{efr}} / \xi_{\mathrm{S}}$, which is a function of the proximity parameter $\gamma_{\mathrm{M}}=\left(\sigma_{\mathrm{S}^{\prime}} \xi_{\mathrm{S}}\right)$ $\left.\sigma_{S^{\prime}} \xi_{S^{\prime}}\right)\left(d_{\mathbf{S}^{\prime}} / \xi_{\mathbf{S}^{\prime}}\right)$. The total rate is the sum of the rate of the reduced gap region and of the proximity layer. The effective trapping volume of the core of an Abrikosov vortex, which is trapped in the superconductor, is a cylinder with radius $R_{\mathrm{eff}} \approx 2.7 \xi_{\mathrm{s}}$ and zero gap.
\end{abstract}

\section{Introduction}

Josephson tunnel junctions can be used as detectors for nuclear particles, phonons and photons over a wide energy range [1]. In the case of X-ray detection the tunnel junction is biased in the quasi-particle tunneling regime. An impacting X-ray photon creates a cascade of hot quasi-particles and phonons that relax to the bandgap. Above the thermal equilibrium quasi-particle density an excess density is created, that typically consists of $2 \times 10^{6}$ quasi-particles for a photon energy of about $6 \mathrm{keV}$ (assuming that all photon energy is converted into excess quasiparticles). The excess quasi-particle density gives rise to an excess tunneling current, which is measured.

In practice, however, a large fraction of the excess quasi-particles may be lost by different processes, before tunneling takes place. An important loss mechanism is the trapping of quasi-particles in regions with a lower energy gap due to energy relaxation. In most cases these trapped quasi-particles cannot tunnel anymore. Such regions with a reduced energy gap may be (1) induced by the proximity effect because of the presence of layers with lower or zero gap adjacent to the superconductor, (e.g. anodized or oxi-

1 On leave from the Institute of Solid State Physics, 142432 Chernogolovka, Moscow, Russia. dized surfaces, the initial growth layer of (sputter-) deposited thin films or normal metal layers); (2) the core of a trapped Abrikosov vortex in the superconducting electrode.

A trapping layer can also be advantageously used to collect the excess quasi-particles from a large volume, as was proposed by Booth [2]. The larger excess quasi-particle density in, and the smaller volume of, the trap produces a signal in the read-out junction placed on the trap, that can be up to two or even three orders of magnitude larger than in the case without a trap. For an X-ray detector, that has to have a high efficiency, a trapping layer is inevitable, because of the large volume of the X-ray photon absorber that is needed.

Although strongly needed for the design of an Xray detector, a detailed study of the trapping effect was lacking up till now. The relaxation rate of quasiparticles from a certain energy down to the bandgap was calculated by Kaplan et al. [3] for the case of a homogeneous, weak-coupling superconductor in thermodynamic equilibrium. In this paper we calculate the effective quasi-particle relaxation rates in a dirty superconductor, that is in thermal equilibrium but has a spatially inhomogeneous order parameter, on the basis of microscopic theory. Two cases are treated:

(1) the reduction of the order parameter in a su- 
perconducting layer $\mathrm{S}$ (with critical temperature $T_{\mathrm{c}} \mathrm{S}$ ) due to the presence of a proximity layer $S^{\prime}$ (with $\left.T_{c} S^{\prime}\right):$ and

(2) the effect of the core of an Abrikosov vortex.

As we are working on the development of X-ray Josephson junction detectors for space application based on the $\mathrm{Nb}\left(T_{\mathrm{c}}=9.2 \mathrm{~K}\right) / \mathrm{Al}\left(T_{\mathrm{c}}=1.3 \mathrm{~K}\right)$ system and operated at ${ }^{4} \mathrm{He}$ temperatures $(T \approx 1 \mathrm{~K})$, the calculations especially apply to that case. However, it can be shown that the critical temperature of the proximity layer enters the problem only very weakly. The relevant parameter is $\log \left(T_{c} \mathrm{~S} / T_{c} \mathrm{~S}^{\prime}\right)$ and its variation can be easily taken into account by means of a simple renormalization procedure [4]. The results will, therefore, also apply fairly well to other combinations of superconductors.

\section{Theory}

The Eliashberg equations for a spatially inhomogeneous superconductor have the general form [5]:

$$
\begin{aligned}
& \text { i) }(\omega, \boldsymbol{x})=\omega Z(\omega, \boldsymbol{x}) \\
& =\omega-\int_{0}^{\infty} \mathrm{d} \omega^{\prime} \operatorname{Re} G\left(\omega^{\prime}, \boldsymbol{x}\right) \int_{0}^{\infty} \mathrm{d} \Omega \alpha^{2}(\Omega) F(\Omega) \\
& \times\left[\frac{f\left(-\omega^{\prime}\right)+n(\Omega)}{\omega^{\prime}+\omega+\Omega+\mathrm{i} \delta}-\frac{f\left(-\omega^{\prime}\right)+n(\Omega)}{\omega^{\prime}-\omega+\Omega-\mathrm{i} \delta}\right. \\
& \left.+\frac{f\left(\omega^{\prime}\right)+n(\Omega)}{-\omega^{\prime}+\omega+\Omega+\mathrm{i} \delta}-\frac{f\left(\omega^{\prime}\right)+n(\Omega)}{-\omega^{\prime}-\omega+\Omega-\mathrm{i} \delta}\right] \\
& \tilde{J}(\omega, \boldsymbol{x})=\Delta(\omega, \boldsymbol{x}) Z(\omega, \boldsymbol{x}) \\
& =\int_{0}^{\infty} \mathrm{d} \omega^{\prime} \operatorname{Re} F\left(\omega^{\prime}, \boldsymbol{x}\right) \int_{0}^{\infty} \mathrm{d} \Omega \alpha^{2}(\Omega) F(\Omega) \\
& \quad \times\left[\frac{f\left(-\omega^{\prime}\right)+n(\Omega)}{\omega^{\prime}+\omega+\Omega+\mathrm{i} \delta}+\frac{f\left(-\omega^{\prime}\right)+n(\Omega)}{\omega^{\prime}-\omega^{\prime}+\Omega-\mathrm{i} \delta}\right. \\
& \left.-\frac{f\left(\omega^{\prime}\right)+n(\Omega)}{-\omega^{\prime}+\omega+\Omega+\mathrm{i} \delta}-\frac{f\left(\omega^{\prime}\right)+n(\Omega)}{-\omega^{\prime}-\omega+\Omega-\mathrm{i} \delta}\right] \\
& -\mu^{*} \int_{0}^{\omega_{\mathrm{c}}} \mathrm{d} \omega^{\prime} \operatorname{Re} F\left(\omega^{\prime}, \boldsymbol{x}\right) \tanh \left(\beta \omega^{\prime} / 2\right)
\end{aligned}
$$

$\Delta(\omega, \boldsymbol{x})$ and $Z(\omega, \boldsymbol{x})$ are the space- and frequency- dependent order parameter and renormalization function, respectively: $G$ and $F$ are the normal and anomalous Green's functions of a superconductor. The function $\alpha^{2}(\Omega) F(\Omega)$ is the spectral weight function of the clectron-phonon interaction, $\Omega$ being the phonon frequency. $\mu^{*}$ is the Coulomb pseudopotential and $\omega_{\mathrm{c}}$ is a cutoff frequency of the order of $\left(\omega_{\mathrm{D}}\right.$. The space coordinates are normalized to the coherence length $\xi_{s}$.

The poles $\epsilon_{p}$ of the single-particle Green's function in the superconducting state are determined by

$\tilde{\omega}^{2}-\epsilon^{2}-\tilde{j}^{2}=0$.

Following the procedure of Kaplan et al. one can introduce an electron scattering rate $\Gamma(\omega, x)$ by setting $\omega=E-\mathrm{i} \Gamma, Z(\omega, \boldsymbol{x})=Z_{1}(\omega, \boldsymbol{x})+\mathrm{i} Z_{2}(\omega, \boldsymbol{x})$, and $J(\omega)$. $\boldsymbol{x})=\tilde{J}_{1}(\omega, \boldsymbol{x})+\mathrm{i} \tilde{J}_{2}(\omega, \boldsymbol{x})$. Assuming that the imaginary parts are small compared to the real parts. one then finds from eq. (2) that $E=\left(\epsilon_{\mathrm{p}}^{2}+\tilde{J}_{1}^{2}\right)^{1 / 2} / Z_{1}$ and

$$
\begin{aligned}
& \Gamma(\omega, x)=\omega Z_{2}(\omega, x) / Z_{1}(\omega, x) \\
& \quad-\tilde{J}_{1}(\omega, x) \tilde{J}_{2}(\omega, x) / Z_{1}^{2}(\omega, x)(\omega) .
\end{aligned}
$$

Here $E$ is the quasi-particle energy and the quantity $\Gamma(\omega, x)$ determines a quasi-particle decay rate:

$\frac{1}{\tau_{\mathrm{sc}}(\omega, x)}=2 \Gamma(\omega, x)$.

We will consider the case of small frequencies ( $)$ compared to phonon frequencies, so that the frequency dependence of $Z_{1}$ and $\tilde{A}_{1}$ can be neglected. Determining the functions $Z$ and $\tilde{J}$ from eq. (1), a generalization of eq. (6) of ref. [3] for $\tau_{\mathrm{Sc}}^{-1}$ in the spatially inhomogeneous case is obtained. For the most interesting case of very low temperatures, $T \rightarrow 0$. the answer is

$$
\begin{aligned}
& \frac{1}{\tau_{\mathrm{SC}}(\omega, \boldsymbol{x})}=\frac{2 \pi}{\hbar Z_{1}(0)} \int_{0}^{\omega-\operatorname{gg}_{(x)}(\boldsymbol{x})} \mathrm{d} \Omega \alpha^{2}(\Omega) F(\Omega) \\
& \times\left[\operatorname{Re} G(\boldsymbol{x}, \omega-\Omega)-\frac{J(\boldsymbol{x})}{\omega} \operatorname{Re} F(\boldsymbol{x}, \omega-\Omega)\right] .
\end{aligned}
$$

This gives the quasi-particle relaxation rate from $\mathrm{cn}$ ergy $\omega$ to $\mathcal{A}_{\mathrm{g}}(\boldsymbol{x})$. Here $\mathcal{A}_{\mathrm{g}}(\boldsymbol{x})$ is the $\boldsymbol{x}$-dependent value of the energy gap of the system and $\Delta(\boldsymbol{x})$ is the real part of the order parameter (which is frequency independent for small $(\omega)$. Because of the limit $T \rightarrow 0$ 
this expression does not contain quasi-particle recombination and corresponds to energy relaxation with rate $\tau_{\mathrm{SC}}^{-1}$ only. In the low-frequency approximation one can write $\alpha^{2}(\Omega) \mathrm{F}(\Omega)=b \Omega^{2}$ and then define a characteristic time $\tau_{0}=Z_{1}(0) \hbar / 2 \pi b\left(k T_{\mathrm{c}}\right)^{3}$ for a given material [3]. Finally, one can define an effective rate in some limited volume of a superconductor by summing the contributions of different regions. For example, in the one-dimensional case one has:

$$
\begin{aligned}
& \left\langle\frac{\tau_{0}}{\tau_{\mathrm{SC}}(\omega, x)}\right\rangle=\frac{1}{L} \int_{0}^{L} \mathrm{~d} x \int_{0}^{\omega-\Delta_{\mathrm{g}}(x)} \mathrm{d} \Omega \Omega^{2} \\
& \quad \times\left[\operatorname{Re} G(x, \omega-\Omega)-\frac{\Delta(x)}{\omega} \operatorname{Re} F(x, \omega-\Omega)\right],
\end{aligned}
$$

where $L$ is the size of a region with reduced energy gap. In eq. (5) and further on, energies are normalized to $k T_{c}$. At low temperatures most of the quasi-particles in the bulk material are near the bandgap and thus one can set $\omega$ equal to the gap energy of the bulk material $\Delta_{\mathrm{O}}$.

\subsection{Proximity layer}

We will apply the above formalism to two different cases which are of immediate interest for the application in Josephson junction X-ray detectors. First we consider the case of a layer with an energy gap that is reduced by the proximity effect due to the presence of a layer with a lower (but non-zero) bulk value of the order parameter, $\Delta_{\mathrm{OS}}$. Secondly, the effective relaxation rate in an Abrikosov vortex is calculated. The spatial dependence of the order parameter in these cases is shown schematically in fig. 1 (a).

For the determination of $\left\langle\tau_{0} / \tau_{\mathrm{sc}}(\omega, \boldsymbol{x})\right\rangle$ one needs to determine the generalized densities of states $\operatorname{Re} G(\omega, \boldsymbol{x})$ and $\operatorname{Re} F(\omega, \boldsymbol{x})$. In a spatially homogeneous superconductor, which is in thermal equilibrium, these are given by the well-known expressions

$$
\begin{aligned}
& \operatorname{Re} G(\omega)=\operatorname{Re} \frac{\omega}{\left(\omega^{2}-\Delta^{2}(\omega)\right)^{1 / 2}}, \\
& \operatorname{Re} F(\omega)=\operatorname{Re} \frac{\Delta(\omega)}{\left(\omega^{2}-\Delta^{2}(\omega)\right)^{1 / 2}} .
\end{aligned}
$$
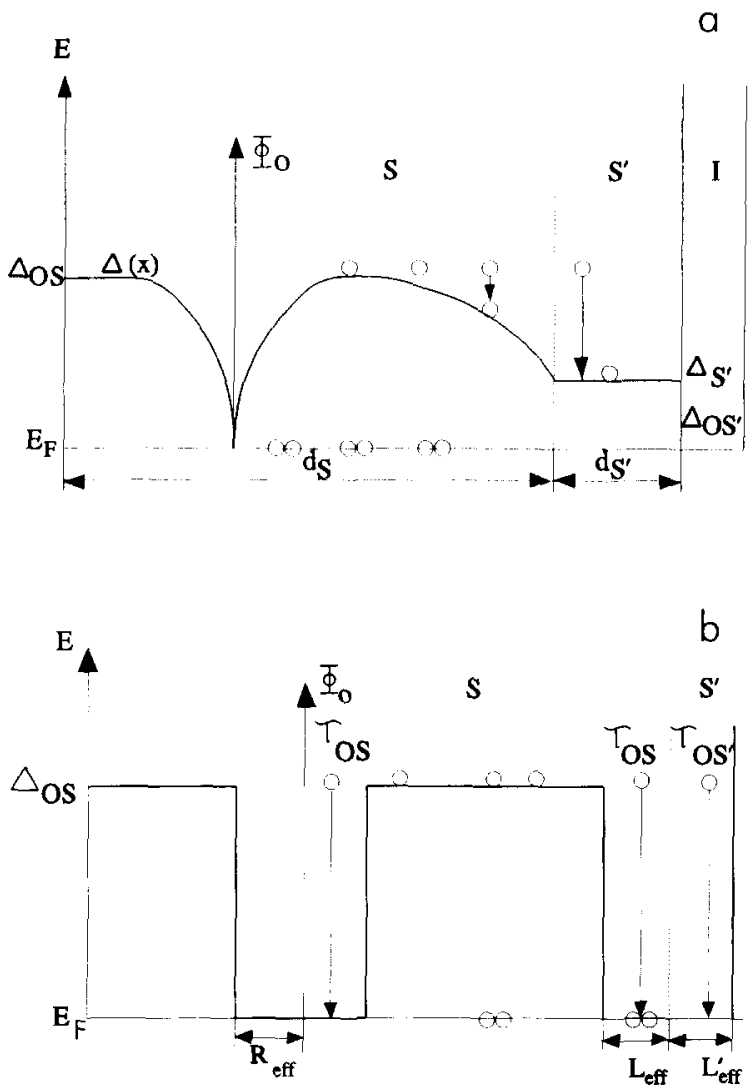

Fig. 1. (a) Schematic space dependence of the order parameter $\Delta(x)$ in a bilayer, due to the presence of a superconducting proximity layer $S^{\prime}$ with bulk gap $\Delta_{0 S^{\prime}}$ and of a trapped Abrikosov vortex in the superconducting layer $S$ with bulk gap $\Delta_{0 s}$. (b) Effective dimensions of the quasi-particle relaxation traps due to the reduced gap regions in the $\mathrm{SS}^{\prime}$-bilayer.

In the general case one should solve the equations of the microscopic theory applying them to any specific model under consideration. In a dirty superconductor the functions $G$ and $F$ obey the so-called Usadel equations [6] which take the form:

$$
\begin{gathered}
\phi\left(\omega_{n}, \boldsymbol{x}\right)=\Delta\left(\omega_{n}, \boldsymbol{x}\right) \\
+\xi_{\mathrm{s}}^{2} \frac{\pi T_{\mathrm{c}}}{\omega_{n} G\left(\omega_{n}, \boldsymbol{x}\right)} \nabla\left[G^{2}\left(\omega_{n}, \boldsymbol{x}\right) \nabla \phi\left(\omega_{n}, \boldsymbol{x}\right)\right], \\
\Delta\left(\omega_{n}, \boldsymbol{x}\right) \ln \frac{T}{T_{\mathrm{c}}}+2 \pi T \sum_{\omega_{n}}\left(\Delta\left(\omega_{n}, \boldsymbol{x}\right) / \omega_{n}\right. \\
\left.\quad-F\left(\omega_{n}, \boldsymbol{x}\right)\right)=0,
\end{gathered}
$$


$\phi=\omega_{n} F / G, \quad G=\left(\omega_{n}\left[\omega_{n}^{2}+\phi^{2}\right]^{-1 / 2}\right.$.

$F=\phi\left[\omega_{n}^{2}+\phi^{2}\right]^{-1 / 2}$,

$\omega_{n}=\pi T(2 n+1), n=0,1,2 \ldots$.

Equation ( $7 \mathrm{~b})$ is the self-consistency relation for the determination of the order parameter $A\left(\omega_{n}, x\right)$. For the calculation of $G$ and $F$ in a $\mathrm{SS}^{\prime}$ sandwich, these equations are coupled by some boundary conditions and therefore have to be solved simultaneously in the $S$ and $S^{\prime}$ layer (in the general case numerically).

We assume that no barrier exists at the interface and $T_{\mathrm{cS}}>T_{\mathrm{cs}} ; d_{\mathrm{S}} \gg \xi_{\mathrm{S}} \gg l_{\mathrm{s}}, l_{\mathrm{S}^{\prime}} \leqslant d_{\mathrm{S}^{\prime}} \ll \xi_{\mathrm{S}^{\prime}}$, i.c., both materials are in the dirty limit. $\left(d_{\mathrm{S}}\left(d_{\mathrm{S}^{\prime}}\right), l_{\mathrm{S}}\left(l_{\mathrm{S}^{\prime}}\right)\right.$, and $\xi_{\mathrm{S}}\left(\xi_{\mathrm{S}^{\prime}}\right)$ are the thickness, the electron mean frec path and the coherence length of the $S\left(S^{\prime}\right)$ layer, respectively.) The last condition also implies that the function $G, F$ and $\Delta$ are assumed to be constant over the $S$ layer. Details on this model of the proximity effect are given in ref. [7] for a SN sandwich $\left(T_{\mathrm{cS}^{\prime}}=0\right)$ and in ref. [8] for the more general case of a SS' sandwich. It was shown that in both cases the extent of the influence of the proximity effect is determined by the parameter

$\gamma_{\mathrm{M}}=\left(\sigma_{\mathrm{S}^{\prime}} \xi_{\mathrm{S}} / \sigma_{\mathrm{S}} \xi_{\mathrm{S}^{\prime}}\right)\left(d_{\mathrm{S}^{\prime}} / \xi_{\mathrm{S}^{\prime}}\right)$.

where $\sigma_{\mathrm{S}, \mathrm{S}^{\prime}}$ are the normal state conductivities of the $S$ and $S^{\prime}$ metals. Generally, the larger the ${ }^{\prime}$ value the stronger is the suppression of the order parameter on the SS' boundary.

In the following we consider the case of a $\mathrm{Nb} / \mathrm{Al}$ sandwich at a temperature of $1 \mathrm{~K}$, thus $T<T_{\mathrm{S}}$. However. it is stressed again that the critical temperature ratio $T_{\mathrm{CS}} / T_{\mathrm{cS}}$ influences the results only very little.

As an illustration, the normalized densities of states $N(\omega, x)=\operatorname{Re} G_{\mathrm{S}}(\omega, x)$ (where $(\omega)=-\mathrm{i}\left(\omega_{n}\right)$ are plotted in fig. 2 for ${ }^{\prime} M=10$ at different distances $x / \xi_{\mathrm{s}}$ from the SS' boundary in the S-region. In the S'-region $\operatorname{Re} G_{S^{\prime}}(\omega)$ takes the value at the boundary Re $G_{\mathrm{S}}(\omega, 0)$. It is seen that the gap value $A_{\mathrm{g}}$ is suppressed relative to the bulk value $A_{0 s}$. The value of $d_{\mathrm{g}}$ is the same for all points in $\mathrm{S}$, the difference being in the values of $N(\omega, x)$ at $A_{\mathrm{g}}<\omega<A_{0 \mathrm{~S}}$, which become small as $x / \xi_{\mathrm{S}} \geqslant 3$.5. This means that a large region of the $\mathrm{S}$ material near the $\mathrm{SS}^{\prime}$ boundary has a reduced gap value $d_{\mathrm{g}}<\Delta_{0 \mathrm{~S}}$. On the other hand the en-

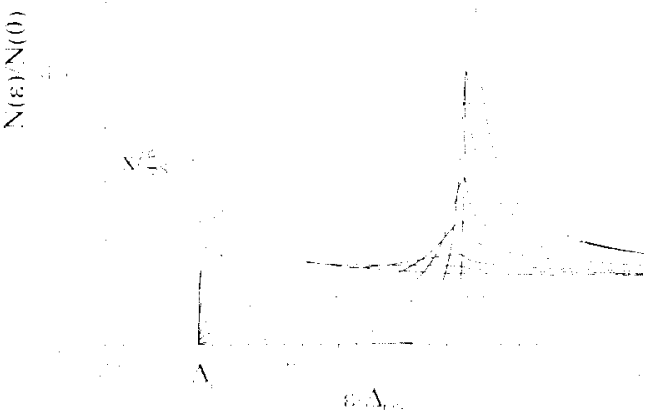

Fig. 2. Normalized quasi-particle densities of states in the S layer at $T \ll T_{\text {cs }}$ and at various distances to the SS'-interface, duc to the proximity effect with an $\mathrm{S}^{\prime}$-layer with $T_{\mathrm{C}}<T_{\mathrm{cS}}$ with $\gamma_{\mathrm{M}}=10$.

ergy gap in the $\mathrm{S}^{\prime}$ layer is increased compared to the bulk value, $J_{\mathrm{g}}>J_{\mathrm{os}}$.

In fig. 3 the quantities $\tau_{0} / \tau_{\text {ses }}(x)$ and $A(x) / \pi k T_{\text {cs }}$ (inset), are plotted to illustrate the relative contribution of different regions of $S$ to the scattering rate (for the particular value ${ }_{i}^{\prime} \mathrm{M}=10$ ). and the behaviour

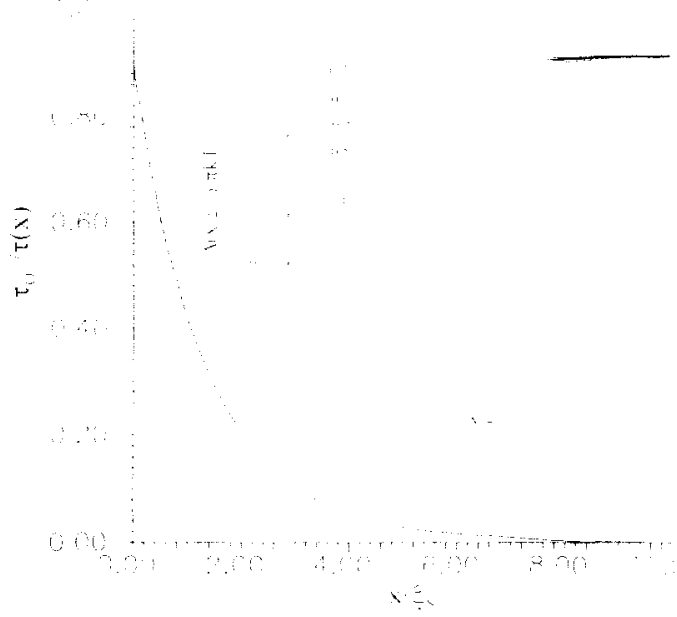

Fig. 3. Normalized relaxation rate in the S-layer at $T \ll T_{c}$ as a function of the distance to the interface of the SS'-layer, due to the proximity effect with an $\mathrm{S}^{\prime}$-layer with $T_{\mathrm{cs}}<T_{\mathrm{cS}}$ with $\gamma_{\mathrm{M}}=10$. Inset: Normalized order parameter in the S-layer as a function of the distance to the SS'-interface for various values for the proximity parameter ${ }^{\prime} \mathrm{M}$. 
of the order parameter $\Delta(x)$ for a number of $\gamma_{M}$ values.

For the presentation of the final result, it is useful to define an effective thickness $L_{\mathrm{eff}}$ of a layer in the $S$ material that is in the normal state $\left(\Delta_{\mathrm{g}}=0\right.$ ) (see also fig. 1(b)), which gives the same total scattering rate as the reduced gap region by the relation

$\int_{0}^{\infty} \frac{1}{\tau_{\mathrm{scS}}(x)} \mathrm{d} x=L_{\mathrm{eff}} \frac{1}{\tau_{\mathrm{scS}}\left(A_{\mathrm{g}}=0\right)}$.

The integral in the left-hand side of eq. (9) is finite, because $\tau_{\mathrm{s}}^{-1}(x) \rightarrow 0$ as $x \rightarrow \infty$, provided $\omega=\Delta_{0 \mathrm{~s}}$. The quantity $\tau_{\mathrm{scS}}^{-1}\left(\Delta_{\mathrm{g}}=0\right)$ can be calculated from eq. (16) of ref. [3] for $\Delta=0, \omega=\Delta_{0 \mathrm{~s}}$ and can be interpreted as the relaxation rate in the $\mathrm{S}$ material in the normal state for quasi-particles with energy equal to $A_{0 \mathrm{~S}}$ down to the Fermi-energy. Taking into account that

$\tau_{\mathrm{scS}}^{-1}\left(\Delta_{\mathrm{g}}=0\right)=\frac{2 \pi b\left(k T_{\mathrm{cs}}\right)^{3}}{Z_{\mathrm{l}}(0) \hbar} \frac{1}{3}\left(\frac{\Delta_{0 \mathrm{~s}}}{k T_{\mathrm{cS}}}\right)^{3} \simeq \frac{1.82}{\tau_{\mathrm{oS}}}$

(making use of the BCS-relation $2 \Delta_{0 \mathrm{~S}} / k T_{\mathrm{cs}}=3.52$ ), the function $L_{\mathrm{efr}}\left(\gamma_{\mathrm{M}}\right)$ for the $\mathrm{S}$ material is determined, using the result of the calculations of $\tau_{0} /$ $\tau_{\mathrm{scS}}(x)$ as shown in fig. 3. An analogous procedure has been applied to the trapping layer $S^{\prime}$ by introducing the length $L_{\text {eff }}^{\prime}$ for the S' material in the normal state (with $\Delta_{0 \mathrm{~S}^{\prime}}=0$ ), which corresponds to the

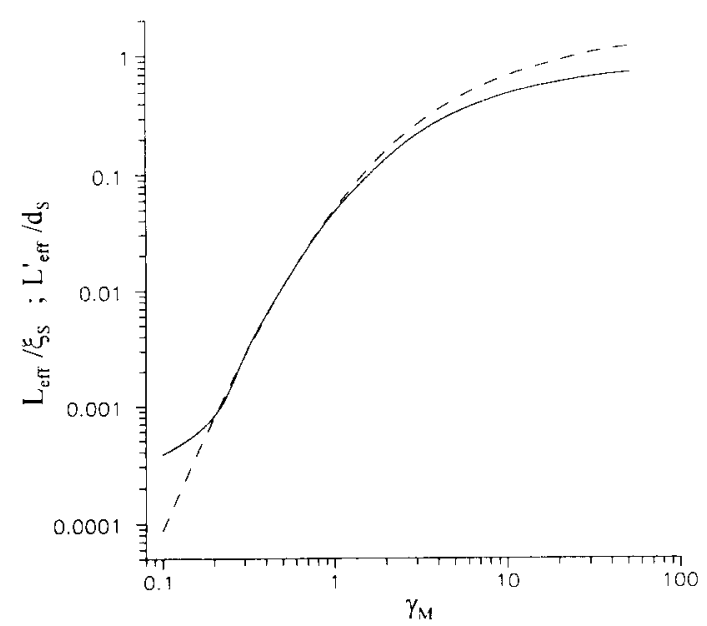

Fig. 4. Normalized effective thickness $L_{\mathrm{eff}} / \xi_{\mathrm{s}}\left(L_{\mathrm{eff}}^{\prime} / d_{\mathrm{S}^{\prime}}\right)$ of the trapping layer in the $S$ - $\left(S^{\prime}\right.$-) layer of a $S^{\prime}$-bilayer as a function of the proximity parameter $\gamma_{\mathrm{M}}$ at temperature $T<T_{\mathrm{cs}}$. given thickness $d_{\mathrm{S}^{\prime}}$ of the $\mathrm{S}^{\prime}$-layer, $d_{\mathrm{S}^{\prime}} / \tau_{\mathrm{scS}^{\prime}}=$ $L_{\text {eff }}^{\prime} / \tau_{\mathrm{scS^{ \prime }}}\left(A_{\mathrm{g}}=0\right)$. In fig. 4 both functions $L_{\mathrm{eff}} / \xi_{\mathrm{S}}\left(\gamma_{\mathrm{M}}\right)$ and $L_{\mathrm{eff}}^{\prime} / d_{\mathrm{S}^{\prime}}\left(\gamma_{\mathrm{M}}\right)$ are presented.

\subsection{Abrikosov vortex}

The second problem to be treated is that of an isolated Abrikosov vortex trapped in the bulk material. The problem of determining an effective relaxation time of a vortex can be solved as follows. First the equations for the Green's functions of a superconductor $G$ and $F$ in the core region of the vortex, where the order parameter is strongly suppressed, are solved. Then the densities of states $\operatorname{Re} G(\omega, \rho), \operatorname{Re}$ $F(\omega, \rho)$ for various distances $\rho$ from the centre of the core are determined. An effective radius $R_{\text {eff }}$ for the trapping region of the core can be defined analogously to eq. (9).

The Usadel equations for the Green's functions of a dirty superconductor in the limit $\kappa \gg 1$, in the magnetic field of a single Abrikosov vortex, are [7]

$$
\begin{aligned}
& \frac{\mathrm{d}^{2} \vartheta}{\mathrm{d} \rho^{2}}+\frac{1}{\rho} \frac{\mathrm{d} \vartheta}{\mathrm{d} \rho}-\omega_{n} \sin \vartheta \\
& \quad-Q^{2}(\rho) \sin \vartheta \cos \vartheta+\Delta\left(\omega_{n}, \rho\right) \cos \vartheta=0, \\
& \Delta \ln \frac{T}{T_{\mathrm{c}}}+2 \pi T \sum_{\omega_{n}}\left(\Delta / \omega_{n}-\sin \vartheta\right)=0,
\end{aligned}
$$

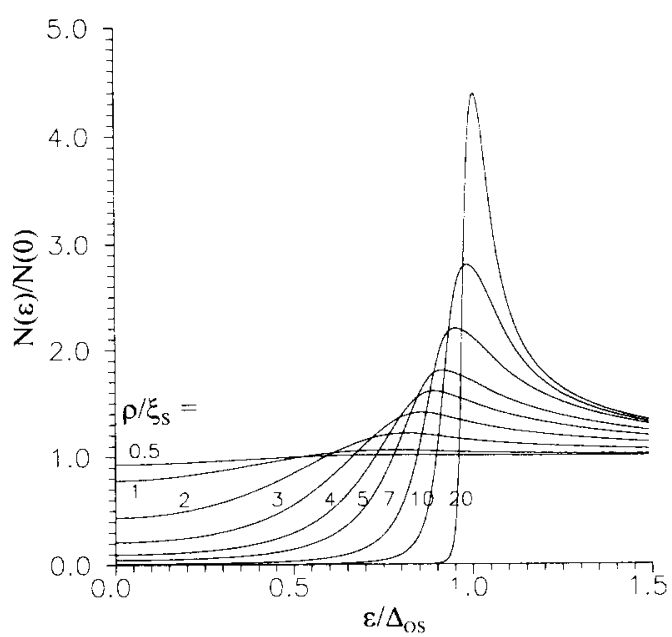

Fig. 5. Normalized quasi-particle densities of states in the $S$ layer at $T \ll T_{\text {cs }}$ due to Abrikosov vortex and at various distances $\rho / \xi_{\mathrm{s}}$ from the centre of the core of the vortex. 
where $F=\sin \vartheta, G=\cos \vartheta$, and the gradient-invariant vector-potential $Q(\rho)=\rho^{-1}$. The boundary conditions read:

$J(p=0)=\vartheta\left(\omega_{n}, 0\right)=0$ :

$\mathrm{d} \Delta(\rho=\infty) / \mathrm{d} \rho=\mathrm{d} \vartheta\left(\omega_{n}, \infty\right) / \mathrm{d} \rho=0$.

The results for the density of states $N(\omega, \rho)=\operatorname{Re}$ $G(\omega, \rho)$ for different $\rho$ values are shown in fig. 5 .

After the calculation of the $\tau_{\mathrm{sc}}^{-1}(\rho)$ distribution and the averaging over the cross-section of the vortex core, one obtains the effective core radius

$R_{\mathrm{eff}} / \xi_{\mathrm{s}} \approx 2.7$.

\section{Discussion}

Now the effective relaxation rate $\tau_{\text {eff }}^{-1}$ due to an $S^{\prime}$ layer with thickness $d_{S^{\prime}}$ and area $A^{\prime}$ on an S-layer. with thickness $d_{\mathrm{S}}$ and area $A$, is calculated. It is assumed that the fraction of time the quasi-particles spend in the trapping area is proportional to the volume fraction of the trap to the total volume of the $\mathrm{S}$ and $\mathrm{S}^{\prime}$ layers [2]. In the case $d_{\mathrm{S}} \gg d_{\mathrm{S}^{\prime}}$ one arrives at an effective rate

$$
\frac{1}{\tau_{\mathrm{eff}}}=\frac{1.82}{d_{\mathrm{S}}}\left[\frac{L_{\mathrm{cff}}}{\tau_{\mathrm{OS}}}+\frac{L_{\mathrm{eff}}^{\prime}}{\tau_{\mathrm{oS} \mathrm{S}^{\prime}}}\right] \frac{A^{\prime}}{A}
$$

where $L_{\mathrm{eff}}$ and $L_{\mathrm{eff}}^{\prime}$ are obtained from fig. 4 . If ${ }_{i m}$ is of order unity then $L_{\mathrm{eff}} \approx L_{\mathrm{eff}}^{\prime}$ and $\tau_{\mathrm{eff}}^{-!}$is mainly determined by the smallest $\tau_{0}$ of the bilayer.

In the case of an $\mathrm{Al}$ proximity layer, the characteristic time $\tau_{0, \mathrm{Al}}(110 \mathrm{~ns})$ is orders of magnitude larger than that of most other metals (e.g. $\tau_{0 . \mathrm{Nh}}=0.15$ ns). This means that the trapping mainly takes place in the reduced gap region of the S-layer and not in the $S^{\prime}$-layer. Secondly, $\mathrm{Al}$ is a very effective material for introducing a substantial reduced gap region by even very thin Al layers, because ${ }^{\prime} M$ becomes large due to the large normal state conductivity $\sigma_{\mathrm{S}^{\prime}}$. As an example, consider a $\mathrm{Nb} / \mathrm{Al}$ junction with $\mathrm{Al}$ thickness $d_{\mathrm{S}^{\prime}}=25 \mathrm{~nm}$ giving $\gamma^{\prime} \approx 1$ (ref. [9]), $1^{\prime}=A$, $\xi_{\mathrm{s}}=40 \mathrm{~nm}$ and $d_{\mathrm{s}}=300 \mathrm{~nm}$. Then the effective time constant is as small as $\tau_{\text {eff }} \approx 13 \mathrm{~ns}$. Even for a $10 \mu \mathrm{m}$ thick $\mathrm{Nb}$ layer, the trapping is still fairly effective: $\tau_{\mathrm{eff}} \approx 0.41 \mu \mathrm{s}$. In junctions with a relatively thin electrode a degraded surface can also create a very ef- fective trapping area and cause large quasi-particle loss [10].

In the case of an Abrikosov vortex, the effective relaxation rate is given by

$$
\frac{1}{\tau_{\mathrm{cff}}}=\frac{\pi R_{\mathrm{eff}}^{2} L_{\phi}}{I_{\mathrm{S}}} \frac{1.82}{\tau_{0 \mathrm{~S}}}=\frac{5 s L_{\phi}}{\tau_{0 \mathrm{~S}} I_{\mathrm{S}}} 42 .
$$

where $L_{\phi}$ is the length of the flux line in the superconductor with volume $l_{S}$ (assuming that the vortex is trapped in the bulk of the superconductor ). In the case of a junction with an electrode with sides of length $L$ and thickness $d_{\mathrm{s}}, \tau_{\mathrm{ctf}}$ is proportional to $L^{2}$ $\left(L d_{\mathrm{S}}\right)$ for a fluxline perpendicular (parallel) to the layer. For a $\mathrm{Nb}$ layer with $d_{\mathrm{S}}=300 \mathrm{~nm}$ and $L=50$ $\mu \mathrm{m}$ this results in $\tau_{\mathrm{eff}^{\prime} \|}=3.3 \mathrm{~ns}$ and $\tau_{\mathrm{eff}} .=0.56 \mu \mathrm{s}$ Because X-ray detectors are normally operated with a field in the plane of the junction barrier in order 10 suppress the Josephson current. trapped vortices may occur without being noticed, except for enhanced quasi-particle losses.

When a trapping layer adjacent 10 the barrier is used as a collector of excess quasi-particles in the absorber, the tunneling rate $\tau_{\text {tun }}^{-1}$ through the barrier is changed due to the proximity effect. The tunneling rate out of a spatially homogeneous superconducting layer with volume $l_{\mathrm{s}}$, through a barrier with normal state resistance $R_{\mathrm{n}}$. is given b! $\tau_{\text {tun }}^{-1}=\left(4 R_{\mathrm{n}} e^{2} l_{\mathrm{s}} N(E)\right)^{-1}$. with $N(E)$ the quasi-parlicle density of states in the superconductor at energ! $E[10.11]$. In the literature it is assumed that the trapping layer can be described by a square well [2] The volume in which the quasi-particles are confined is then cqual to $I_{\text {trap }}=l^{\prime} d_{S^{\prime}}$ and one assumes a spatially independent density of states in the well: $N(E)=N_{\mathrm{n}}\left(E_{\mathrm{F}}\right) \operatorname{Re}(i(E)$ given by eq. (6). The total rate is then assumed to be equal to the sum of the rates out of the trap and out of the bulk layer. However. this procedure does not take into account the existence of a region in the absorber layer with a spatially varying reduced gap. Consequently the volume, to which the quasi-particles are confined. depends on the quasi-particle energy. As has been shown above, the density of states in the trapping region is also spatially dependent and differs strongly from that in the bulk. A more detailed calculation of the effective tunneling rate is therefore necessary and is the subject of further study. 


\section{Conclusions}

The quasi-particle relaxation rates in reduced gap regions of a dirty superconductor at low temperatures, due to (1) the proximity effect with a layer with lower critical temperature, and (2) the gap reduction caused by a trapped Abrikosov vortex, were determined from microscopic theory. In the first case the reduced gap regions can be characterized by effective lengths which are a function of the proximity parameter $\gamma_{M}$, assuming an order parameter equal to zero in the trap. The reduced gap region caused by the vortex is characterized by an effective radius $R_{\text {eff }} \cong 2.7 \xi_{\text {s. }}$.

\section{Acknowledgements}

The authors gratefully acknowledge helpful discussions with H. le Grand, P. de Korte, H. Gijsbertsen and J. Flokstra. This work is part of the Superconducting $\mathrm{X}$-ray Detector project, founded by Stichting Fundamenteel Onderzoek der Materie (FOM).

\section{References}

[1] G.H. Wood and B. White, Appl. Phys. Lett. 15 (1969) 237; W. Eisenmenger, in: Physical Acoustics, eds. W.P. Mason and R.N. Thurston (Academic, New York, 1976) Vol. XII, p. 79 ;

J.J. Chang and D.J. Scalapino, Phys. Rev. B 15 (1977) 2651.

[2] N.E. Booth, Appl. Phys. Lett. 50 (1987) 293.

[3] S.B. Kaplan, C.C. Chi, D.N. Langenberg, J.J. Chang, S. Jafarey and D.J. Scalapino, Phys. Rev. B 14 (1976) 4854.

[4] A.A. Golubov, M.Yu. Kupriyanov and S. Polonsky, submitted to JETP.

[5] J. Rammer, Phys. Rev. B 36 (1987) 5665.

[6] K. Usadel, Phys. Rev. Lett. 25 (1970) 560.

[7] A.A. Golubov and M.Yu. Kupriyanov, J. Low Temp. Phys. 70 (1988) 83.

[8] A.A. Golubov and E.P. Houwman et al., to be published.

[9] E.P. Houwman, J.G. Gijsbertsen, J. Flokstra, H. Rogalla, J.B. le Grand, P.A.J. de Korte and A.A. Golubov, Proximity Effect in $\mathrm{Nb} / \mathrm{Al}$, Alox, $\mathrm{Al} / \mathrm{Nb}$ Josephson Tunnel Junctions, presented at the Applied Superconductivity Conference (ASC'92), Chicago, 1992.

[10] P.A.J. de Korte, M.L. van den Berg, M.P. Bruyn, M. Frericks, J.B. le Grand, J.G. Gijsbertsen, E.P. Houwman and J. Flokstra, Superconductive Tunnel Junctions for X-Ray Spectroscopy, presented at the SPIE (The International Society for Optical Instrumentation ) conference, San Diego, 1992.

[11] D.M. Ginsberg, Phys. Rev. Lett. 8 (1962) 204. 